\title{
On Even Matroids
}

\author{
W. T. Tutte* \\ Professor of Mathematics, University of Waterloo, Ontario, Canada \\ (November 16, 1966)
}

\begin{abstract}
This article is intended as a supplement to an earlier paper entitled "Lectures on matroids."
The author takes this opportunity to correct some errors in "Lectures on Matroids." Theorems 4.31 and 4.372 are valid only for binary matroids, the plane of 4.281 must be connected, and the word "reductions" is used in 3.48 instead of "contractions."
\end{abstract}

Key Words: Binary, bridge-separable, even, graphic, matroid.

It is shown in $[1]^{1}$ that every graphic matroid is regular ([1], 5.63) and even ([1], 9.23). Moreover a regular matroid can be characterized as a binary one which has no minor of either of the types called BI and BII. ([1], 7.51). In the present paper we establish a converse theorem: any even matroid which has no minor of Type BI must be graphic.

1. Let $Y$ be an atom of a binary matroid $M$, and suppose it to have the following properties.

(i) $Y$ is bridge-separable

(ii) If $B$ is any bridge of $Y$ in $M$, then $\mathrm{M} \times(\mathrm{B} \cup \mathrm{Y})$ is graphic.

Then $M$ is graphic.

Proof. If possible choose $Y$ and $M$ so that the theorem fails, and $M$ has the least number of cells consistent with this condition.

Clearly there must be at least two bridges of $Y$ in $M$. Since $Y$ is bridge-separable we can arrange these bridges in two non-null disjoint classes $P$ and $Q$ so that no two members of the same class overlap. Let $U_{P}$ be the union of the members of $P$, and let $U_{Q}$ be defined analogously.

Now $Y$ is an atom of $M \times\left(U_{P} \cup Y\right)$. Moreover the bridges of $Y$ in $M \times\left(U_{P} \cup Y\right)$ are the members of $P$, and each determines the same partition of $Y$ as in $M$. ([1], 8.53). Hence $Y$ is totally bridge-separable in $M \times\left(U_{P} \cup Y\right)$. It follows, by the choice of $Y$ and $M$ that $M \times\left(U_{P} \cup Y\right)$ is graphic. Similarly $Y$ is totally bridge-separable in $M \times\left(U_{Q} \cup Y\right)$, and this matroid is graphic.

We may now repeat the argument in the latter part of the proof of ([1], 9.41), with $U_{P}=S$ and $U_{Q}=T$. We thus find that $M$ is graphic.

This contradiction establishes the theorem.

*An invited paper. Present address: University of Waterloo, Ontario, Canada

${ }^{1}$ W. T. Tutte, Lectures on Matroids, J. Res. NBS 69B (Math. and Math. Phys.), Nos. 1 \& 2, 1-47 (1965).
2. Let $Y$ be an atom of a binary matroid $M$ on a set $E$. Let $W$ be an atom of $M \cdot(E-Y)$, on a bridge $B$ of $Y$ in $M$, determining a partition $\{S, T\}$ of $Y$ such that $\mathrm{T} \epsilon \pi(\mathrm{M}, \mathrm{B}, \mathrm{Y})$. Let $\mathrm{Y}_{1}$ denote the atom $\mathrm{S} \cup W$ of $\mathrm{M}$, and write $\mathrm{M}_{1}=\mathrm{M} \times(\mathrm{B} \cup \mathrm{Y})$.

Then $T$ is a bridge of $Y_{1}$ in $M_{1}$. Moreover every other bridge of $Y_{1}$ in $M_{1}$ is also a bridge of $Y_{1}$ in $M$.

Proof. The set $Y \cup W=Y_{1} \cup T$ is a line of $M$ on the flat $B \cup Y$. Hence $T$ is an atom of

$$
(M \times(B \cup Y)) \cdot\left((B \cup Y)-Y_{1}\right)
$$

by ([1], 8.12). It is thus a subset of some bridge $B_{T}$ of $Y_{1}$ in $M_{1}$.

Let $C$ be any bridge of $Y_{1}$ in $M_{1}$. Suppose there is an atom $Z$ of $M \cdot\left(E-Y_{1}\right)$ which meets $C-T$.

There is an atom $X$ of $M$ such that $X \cap\left(E-Y_{1}\right)=Z$.

There is an atom $Z_{1}$ of $M \cdot(E-Y)$ such that

$$
Z_{1} \subseteq X \cap(E-Y) .
$$

Since $C-T$ is a subset of $E-Y$ we may choose $Z_{1}$ to meet $C-T$, by ([1], 1.11). It then follows that $Z_{1}$ is on the bridge $B$ of $Y$ in $M$.

There is an atom $X_{1}$ of $M \times(B \cup Y)$ such that

$$
X_{1} \cap B=Z_{1} .
$$

There is an atom $Z_{2}$ of $(M \times(B \cup Y)) \cdot\left((B \cup Y)-Y_{1}\right)$ such that $Z_{2} \subseteq X_{1} \cap\left((B \cup Y)-Y_{1}\right)$. By ([1], 1.11) we may choose $Z_{2}$ to meet $C-T$. We note that $Z_{2}$ is an atom of $\left(M \cdot\left(E-Y_{1}\right)\right) \times\left((B \cup Y)-Y_{1}\right)$, by ([1], 3.334), and therefore of $M \cdot\left(E-Y_{1}\right)$. Further, $Z_{2}$ is on the bridge $C$ of $Y_{1}$ in $M_{1}$. We thus have

$$
\begin{gathered}
Z_{2} \subseteq X_{1} \cap C=Z_{1} \cap C \\
\subseteq X \cap(E-Y) \cap C \subseteq X \cap(C-T),
\end{gathered}
$$


since

$$
\begin{gathered}
T \cap(E-Y)=\phi, \\
\subseteq\left(Z \cup Y_{1}\right) \cap(C-T)=Z \cap(C-T) .
\end{gathered}
$$

Applying Axiom I to $M \cdot\left(E-Y_{1}\right)$ we deduce first that $Z_{2}=Z$ and then that $Z \subseteq C-T$. Thus $C-T$ is a separator of $M \cdot\left(E-Y_{1}\right)$. $M_{1}$.

We deduce that $B_{T}=T$. Thus $T$ is a bridge of $Y_{1}$ in

Suppose $C$ is another bridge of $Y_{1}$ in $M_{1}$. The above result shows that $C(=C-T)$ is a separator of $M \cdot\left(E-Y_{1}\right)$.

To complete the proof we observe that

$$
\left(M \cdot\left(E-Y_{1}\right)\right) \times C
$$

is connected. For it is identical with

$$
\left(\left(M \cdot\left(E-Y_{1}\right)\right) \times\left((B \cup Y)-Y_{1}\right)\right) \times C,
$$

that is with $\left(M_{1} \cdot\left((B \cup Y)-Y_{1}\right)\right) \times C$ by $([1], 3.333)$, and $C$ is a bridge of $Y_{1}$ in $M_{1}$. Hence $C$ is an elementary separator of $M \cdot\left(E-Y_{1}\right)$, that is a bridge of $Y_{1}$ in $M$.

3. Let $\mathrm{M}$ be any even matroid which has no minor of Type BI. Then $\mathrm{M}$ is graphic.

Proof. Assume that $M$ is not graphic.

If $Y$ is any atom of $M$ there is a bridge $B$ of $Y$ in $M$ such that $M \times(B \cup Y)$ is not graphic, by Theorem 1 . Choose such a $Y$ and $B$ so that $B$ has the least possible number of cells.
Choose $T \epsilon \pi(M, B, Y)$. Now $B$ is nontrivial since $M \times(B \cup Y)$ is nongraphic. Hence there is an atom $Z$ of $M \cdot(E-Y)$ on $B$ determining the partition $\{T, Y-T\}$ of $Y$, by ([1], 8.62). By the definition of this partition the set $Y_{1}=(Y-T) \cup Z$ is an atom of $M \times(B \cup Y)$.

One of the bridges of $Y_{1}$ in $M \times(B \cup Y)$ is $T$, by Theorem 2. Let the others, if any, be enumerated as $C_{1}, C_{2}, \ldots, C_{k}$. The matroid $M \times\left(T \cup Y_{1}\right)$ has rank 2, by $([1], 8.12)$. It is therefore graphic, by ([1], 9.41). Moreover any $C_{i}$ is a bridge of $Y_{1}$ in $M$, by Theorem 2 . Hence $M \times\left(C_{i} \cup Y_{1}\right)$ is graphic, by the choice of $Y$ and $B$. But $M \times(B \cup Y)$ is nongraphic. It follows from Theorem 1 that $Y_{1}$ is not bridge-separable in $M \times(B \cup Y)$.

Now each $C_{i}$ determines the same partition of $Y_{1}$ in $M$ as in $M \times(B \cup Y)$, by ([1], 8.53). Hence if $C_{i}$ and $C_{j}$ overlap as bridges of $Y_{1}$ in $M \times(B \cup Y)$ they overlap also as bridges of $Y_{1}$ in $M$.

Since $Y \cup Z=Y_{1} \cup T$ is a line of $M$ the set $T$ is an atom of $M \cdot\left(E-Y_{1}\right)$, by $([1], 8.12)$. Hence there is a bridge $D$ of $Y_{1}$ in $M$ such that $T \subseteq D$. This bridge is distinct from each of the bridges $\overline{C_{i}}$. Each of the sets $Z$ and $Y-T$ is either null or a union of members of $\pi\left(M, D, Y_{1}\right)$, by the definition of this partition. Hence if $T$ and $C_{i}$ overlap as bridges of $Y_{1}$ in $M \times(B \cup Y)$, then $D$ and $C_{i}$ overlap as bridges of $Y_{1}$ in $M$. For $\pi\left(M \times(B \cup Y), T, Y_{1}\right)=\{Z, Y-T\}$.

From the foregoing results we deduce that since $Y_{1}$ is not bridge-separable in $M \times(B \cup Y)$ it is also not bridge-separable in $M$. But this contradicts the definition of $M$ as an even matroid. The theorem follows.

(Paper 71B4-245) 